\title{
Un projet de développement local en santé : le cas de la MRC Robert-Cliche et de sa coopérative de santé, ou comment une communauté en difficulté peut s'engager dans un processus d'empowerment
}

\author{
Sabrina Tremblay ${ }^{\mathrm{a}}$
}

RÉSUMÉ. L’accès aux services de santé dans les régions rurales ou périphériques fait partie des enjeux de développement important, notamment pour ce qui est de la rétention de la population. Or, on constate que l'État central n'est pas capable de garantir un accès équitable pour tous. Dans le but de renverser cette tendance, certaines collectivités ont mis sur pied une coopérative de santé. Ce type de projet de développement local est alors associé au développement du pouvoir d'agir communautaire, car il témoigne de la capacité des milieux à agir et décider selon leur propres choix et besoins. Afin de mieux comprendre ce processus dans toutes ses subtilités, nous avons procédé à une étude de cas unique, soit celui de la MRC Robert-Cliche et de sa coopérative de santé du même nom. À partir de cette étude, nous avons été en mesure d'identifier plus clairement les quatre dimensions du processus d'empowerment et les interactions qui en découlent.

\begin{abstract}
Access to health services in rural orperipheral regions is one of the major development concerns, particularly regarding population retention. However, it was possible to observe that the central State cannot guarantee equal access for all. In an effort to reverse this trend, some communities have set up a health cooperative. This type of local development project is associated with community empowerment, because it shows the ability of communities to act and decide according to their choices and needs. To better understand this process in all of its many aspects, a unique case study was conducted on the MRC Robert-Cliché organization and its health cooperative. This study enabled to identify more clearly the four dimensions of the empowerment process and the resulting interactions.
\end{abstract}

\section{Introduction}

Depuis les années 1980, le Canada vit des problèmes importants avec ses services de santé de première ligne, ce qui se traduit au Québec par un accès difficile aux médecins de famille (Fédération des médecins omnipraticiens du Québec, 2013). Ces difficultés, dans les régions du Québec, sont parfois vécues très durement par les collectivités éloignées ou périphériques qui rivalisent de stratégies afin d'attirer sur leur territoire des jeunes familles. Malgré tous les attraits que peuvent avoir ces milieux, on sait que le manque d'accès à certains services de proximité clés (éducation, santé, services sociaux) rebute généralement les candidats à venir s'installer dans ces régions. Pour le développement de ces dernières, il va sans dire que c'est une situation particulièrement incapacitante (Simard, 2005).

À ce propos, on sait que les coopératives de santé (CS) du Québec sont justement apparues au courant des années 1990 afin de pallier cette difficulté vécue d'abord et avant tout à l'échelle locale (Girard, 2012). Les CS ont comme objectif d'impliquer les membres d'une communauté dans la production de services de santé afin de favoriser, promouvoir ou maintenir la santé des individus et les

a Professeure, Département des sciences humaines et sociales, Université du Québec à Chicoutimi 
conditions qui lui sont favorables (Brassard et Darou, 2012). Ces cas sont intéressants à étudier, car leur développement s'est fait au départ malgré l'État qui n'est pas habitué à voir d'autres acteurs s'inviter dans la gestion ou la production des services de santé. Plusieurs fois, les porteurs de projet se sont fait dire par les fonctionnaires de l'État : «non, ce n'est pas écrit dans la réglementation, ça ne s'est jamais vu, ça ne peut donc pas se faire. C'est impossible» (Boivin et Fillion, 1999, p. 5). Or, avec l'amplomb qui caractérise ceux qui croient à leur projet, ils ont persévéré. En ce sens, certains auteurs n'ont pas manqué d'associer ces projets à la densification du capital social ou de façon plus large, à l'empowerment communautaire (Brassard et Darou, 2012; Girard, 2011).

L'appropriation du pouvoir par les collectivités locales n'est pas une préoccupation nouvelle dans les sciences du développement régional. D'ailleurs, plusieurs des politiques de développements régionales anciennes et actuelles avaient entre autre comme objectif de soutenir ce processus : la naissance des MRC dans les années 1970, le développement des Sociétés d'aide au développement des collectivités (SADC) et plus tard des Centres locaux de développement (CLD), pour ne nommer que ceux-ci, témoignent tous du désir de l'État central de permettre aux milieux de se prendre en main (Levy, 2012). Or, là aussi, les nombreuses expériences sur le terrain démontrent que le processus d'empowerment communautaire n'est pas simple et qu'il est surtout mal compris. Il est entouré de nombreuses contraintes et enjeux, ce qui laisse à penser que le seul souhait de voir un milieu se prendre en main par une réorientation des ressources exogènes n'est pas elle seule suffisante (Friedman, 1992; Maynard, Gilson et Mathieu, 2012; Mendell, 2006; Ninacs, 2008; Riger, 1993). Si on veut continuer à promouvoir cette approche de développement, il faut clairement s'attarder à bien en comprendre les différentes dimensions et ses mécanismes de fonctionnement (Bacqué, 2006).

C'est ainsi que le phénomène des coopératives de santé québécoises nous est apparu comme un terrain prometteur afin d'étudier plus en détails le processus d'empowerment communautaire. Et pour cause, le secteur de la santé et des services sociaux québécois est en effet typiquement très centralisé, ce degré fluctuant au gré des multiples réformes de la santé inachevées qui se sont succédé
(Vaillancourt, 2017). Au surplus, il est traversé par des enjeux économiques, politiques et corporatistes importants (Bernier, 2011) qui dépassent parfois de beaucoup la capacité du local à agir selon ses propres choix et besoins. C'est donc dans ce contexte que nous exposerons le cas de la coopérative de santé de la MRC Robert-Cliche (Beauce, Québec) qui semble être à l'heure actuelle le projet du genre le plus abouti des 20 dernières années. Cette coopérative a également pu bénéficier d'un appui externe au secteur de la santé, mais qui a été crucial pour son développement : la politique nationale de la ruralité (PNR). Mais avant de se déplacer dans cette petite collectivité beauceronne, il nous semble opportun de nous pencher d'abord sur le concept phare de notre étude, soit celui de l'empowerment communautaire.

\section{L'empowerment}

Empowerment, développement du pouvoir d'agir, capacitation, agencéité... nombreux sont les termes qui ont foisonné ces dernières décennies dans le but de mieux circonscrire le processus d'empowerment. Or, parler d'empowerment, c'est d'abord et avant tout parler de pouvoir. Sans entrer dans toutes ses subtilités, car tel n'est pas l'objet du présent texte, on considère généralement que le pouvoir est une capacité individuelle ou collective de "contrôler les termes d'une relation d'échange afin qu'elle lui soit favorable » (Rui, 2011, p. 85). Il permettrait d'agir malgré les difficultés, l'opposition ou les résistances rencontrées (Weber, 1971), voire, dans certains cas, de manipuler les gens de manière à leur faire faire des choses contraires à leur propre intérêt (Lukes, 2005). Ceci dit, pour Foucault (1975), le pouvoir est présent partout et il se dissémine par le discours (comment on parle de quelque chose) et non par les structures (ex. le gouvernement). On retiendra de ces différents auteurs deux choses: d'abord, les points de vue tombent généralement quelque part entre une vision pluraliste du pouvoir (tout le monde peut avoir du pouvoir) ou élitiste (une minorité dirigeante détient le pouvoir). Deuxième chose, peu importe l'approche utilisée, parler de pouvoir c'est aussi parler de résistance et de contrepouvoir (Rui, 2011). Ce dernier aspect est d'ailleurs l'un des fondements du concept de l'empowerment. 


\subsection{Définitions et conditions d'émergence}

Dans une étude précédente (Tremblay, 2017), nous avons soulevé toute la difficulté de non seulement définir ce terme, mais aussi de comprendre comment il se réalise. À l'heure actuelle, il n'existe que très peu de cadres théoriques qui permettent de bien expliciter ce processus, aussi nous avons choisi de nous tourner vers celui de Ninacs (2008) qui semble être le plus complet en la matière. Bien qu'imparfait, il nous fournit des dimensions de l'empowerment sur lesquelles se baser.

Commençons par la définition. Pour cet auteur, l'empowerment communautaire (EC) est un « état où la communauté est capable d'agir en fonction de ses propres choix et où elle favorise le développement du pouvoir d'agir de ses membres » (Ninacs, 2008, p. 39). Cette définition sous-tend que le processus de développement du pouvoir d'agir ne cherche pas seulement à doter la communauté locale, en tant que tout, d'une capacité autonome d'agir et de décider, mais aussi, qu'elle met en place un ensemble de facteurs structurants qui favorisent le développement de l'agir individuel. Bref, la communauté n'est pas seulement un territoire vivant, c'est aussi un outil de développement social, un peu à l'image que ce que Wilkinson (1991) avait proposé en parlant de ses champs interactionnels communautaires. En développement local, l'EC est souvent vu comme un processus qui permet à une collectivité de se sortir de situation de dépendance face au soutien ou dans certains cas, aux contraintes de l'État. Plus précisément, on parle de «solution de rechange au courant dominant ", de "recherche active d'influence sur les décisions » et de « maîtrise sur les ressources » de la communauté, c'est-à-dire de la capacité d'en disposer, voire de les partager dans une dynamique partenariale (Friedman, 1992; Klein et Champagne, 2011; Ninacs, 2008). Ce dernier point est important, car il suggère que la coordination des différentes forces vives du milieu, couplée aux ressources exogènes, sont nécessaires afin d'entamer et de réaliser un processus d'empowerment (Brennan, Micheal et Cantrell, 2014; Fellin, 2001; Ninacs, 2008).

L'EC ne peut se faire qu'à certaines conditions: d'abord, il faut de «l'espace», c'est-à-dire une possibilité de le développer et de le maintenir. Ensuite, il faut créer ou consolider, selon le cas, un dispositif de participation formel (ex : table de concertation, comité de bénévoles reconnus, assemblée générale des membres, etc.) afin de favoriser les échanges et les apprentissages. Enfin, il doit y avoir mise en réseau des acteurs qui proviennent de la sphère publique, civile et économique de la communauté (Mendell, 2006). Fait à noter, l'empowerment est soumis aux forces politiques et économiques dominantes. Ainsi, l'État peut devenir un obstacle majeur si l'initiative locale va à l'encontre de ses orientations ou politiques publiques (Friedman, 1992; Klein et Champagne, 2011). Malgré tout, l'État connait généralement ses limites et cherche, dans une certaine mesure, à les dépasser (Mendell, 2006). C'est donc à son avantage d'apprendre à traiter avec des communautés en situation de pouvoir et c'est d'ailleurs ce qu'il a fait ces dernières décennies avec son soutien au développement local et à l'économie sociale (Lévesque, 2007). L'enjeu actuel est donc de continuer à favoriser la cohabitation entre les deux logiques d'action, celle de l'État et celle des collectivités en quête de pouvoir sur leur développement.

Cela étant dit, pour Ninacs (2008), le développement du pouvoir d'agir ne peut se mettre en branle que s'il y a un état de perte de pouvoir préalable (disempowerment). Partant du principe que le pouvoir des communautés est une ressource courante, mais qu'il est disséminé un peu partout dans les organisations et les groupes d'intérêts locaux (vision pluraliste du pouvoir), on comprend que la perte de pouvoir communautaire réside surtout dans le fait que ledit milieu est incapable de se coordonner et de se concerter sur des objectifs qui lui permettraient de se lancer dans une action de résilience (Brennan et Israel, 2013). Or, Ninacs (2008) ne propose pas de définition concrète du disempowerment. En fonction de ce que nous venons de préciser, et en gardant à l'esprit qu'une communauté en situation de pouvoir favorise le développement du pouvoir d'agir de ses membres (Ninacs, 2008), nous estimons qu'une communauté disempowered est :

Un milieu aux prises avec plusieurs difficultés sociales, économiques et environnementales, difficultés qui par un effet complexe d'accumulation et d'amplification expliquent aussi l'incapacité dudit milieu à reprendre le contrôle de son développement et lui permettre ainsi de constituer un milieu de vie favorisant le bien-être et la qualité de vie des individus qui le composent (Tremblay, 2017, p. 115). 


\subsection{Les dimensions de l'empowerment}

Comment se réalise l'empowerment communautaire? Pour Ninacs (2008), le processus se réalise sur quatre plans qui se croisent et s'entrecroisent pour former l'EC, un peu à la manière des brins d'une corde.

La première composante est celle de la participation des membres de la communauté. La participation doit être ouverte à tous (et promue comme telle), soutenue par des dispositifs adaptés à la population et leurs besoins et finalement significative en termes d'impacts et de changements possibles. La seconde composante de l'EC concerne les compétences, c'est-à-dire les forces du milieu qui sont le plus souvent personnifiées par des organisations. Ces compétences doivent être reconnues, mais surtout, elles doivent être capables de fonctionner en réseau afin de créer des synergies. La troisième composante est celle de la communication. L'auteur réfère ici aux interactions libres et positives entre les membres de la communauté, c'est-à-dire un climat de libre expression, une transparence dans les processus décisionnels et une circulation efficace de l'information dans la communauté. Enfin, la quatrième et dernière composante est le capital communautaire. Pour, Ninacs (2008), cette composante se traduit par deux éléments. D'abord, celui d'un réservoir (d'où le terme «capital») de sentiments positifs envers sa propre communauté, ce qui pourrait être traduit par le sentiment d'appartenance, et celui de la conscience citoyenne, laquelle réfère au désir d'action engendré par un regard critique et un sentiment de devoir agir pour le bien de sa collectivité (Figure 1).

Soulignons au final que le développement du pouvoir d'agir communautaire est en lien avec les deux autres niveaux d'empowerment soit celui individuel (EI) et aussi celui organisationnel ${ }^{1}$ (EO) que nous ne présenterons pas ici par souci de concision. Pour résumer grossièrement, l'empowerment communautaire (EC) se met en branle quand des individus se rassemblent au sein d'une organisation servant à agir sur une problématique identifiée dans le milieu. Cette organisation doit apprendre à travailler en réseau avec les autres forces du milieu, contribuant par là même au développement de la communauté (Figure 1).

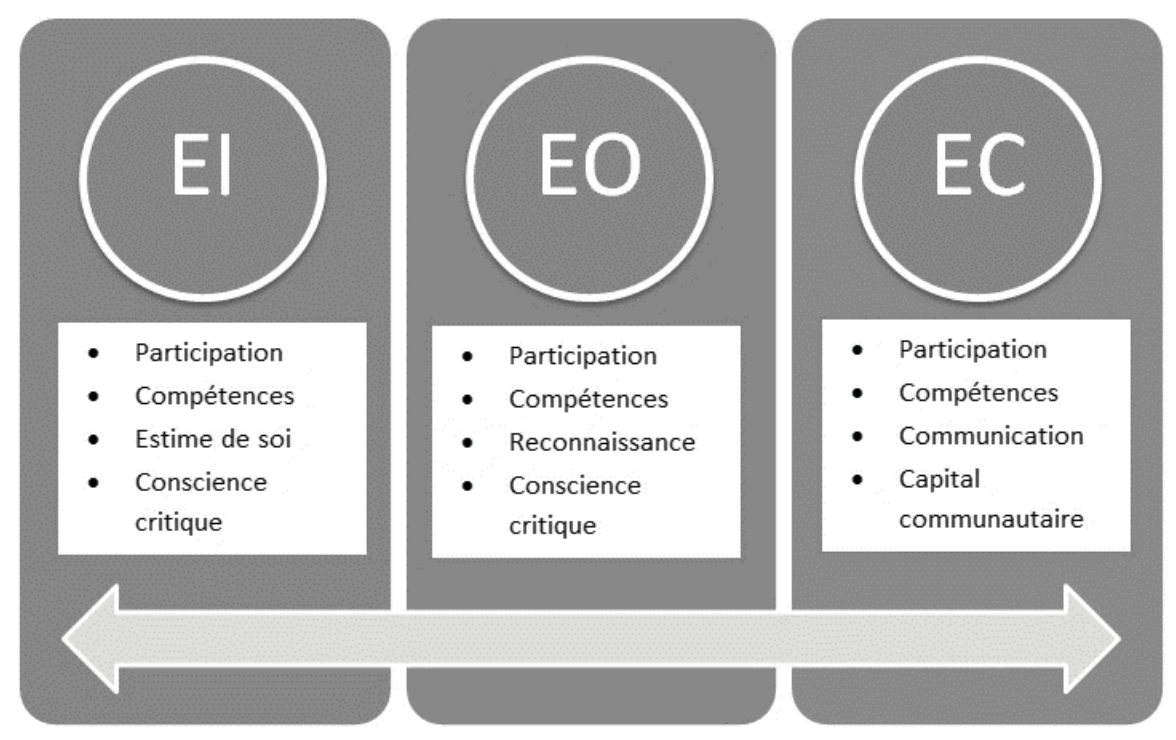

Figure 1- Le processus global d'empowerment, adapté de Ninacs (2008) 


\section{Le cas de la MRC Robert-Cliche et de sa coopérative de santé}

Entre 2013 et 2017, nous nous sommes penchés sur le cas de la CS Robert-Cliche en réalisant une étude de cas unique (Yin, 2009). Cette approche méthodologique nous semblait la plus indiquée en raison de notre désir de comprendre en profondeur le processus d'empowerment communautaire que nous soupçonnions de s'y dérouler. Également, c'était à l'époque la seule CS à desservir une MRC en entier, plutôt qu'une municipalité comme c'était la norme au milieu des années 2000 (Brassard et Darou, 2012), ce qui faisait d'elle d'emblée un cas unique. Pour ce faire, nous avons réalisé une trentaine d'entretiens de recherche auprès d'acteurs clés du milieu (fondateurs, élus, organisations externes, membres de la coopérative), nous avons analysé le corpus documentaire disponible sur le cas, en plus de faire de nombreuses heures d'observation sur le terrain afin de mieux comprendre les dynamiques locales. Toutes les données de recherche ont été gérées et analysées à l'aide du logiciel Nvivo 11.

\subsection{Présentation de la MRC Robert-Cliche}

La MRC Robert-Cliche est une petite communauté rurale d'à peine 20000 habitants. Située dans la région administrative de Chaudière-Appalaches et dans la région culturelle de la Beauce, cette localité a vécu, depuis 1996, plusieurs bouleversements de nature socioéconomique qui l'on conduite à vivre une situation de perte de pouvoir. Afin de bien comprendre les éléments qui ont mené la MRC Robert-Cliche dans sa situation de disempowerment, nous allons nous attarder dans un premier temps à ses particularités locales.

La MRC Robert-Cliche a toujours eu un développement économique plus difficile que ses voisines. $\mathrm{Ne}$ comportant aucun grand centre urbain, ce milieu vit tous les désavantages de la périphérie: fuite des commerces, des capitaux et des familles vers les milieux mieux pourvus en services de tout acabit. Un informateur du milieu l'a résumé très simplement en ces quelques mots : «Ici, tout ça va au nord [vers la ville de Québec] ou au sud [vers la ville de Saint-Georges-de-Beauce] ».
Pourtant, de par sa proximité géographique avec la frontière américaine ainsi que grâce à la fibre entrepreneuriale légendaire des Beaucerons (Palard, 2009), la MRC avait réussi à développer différents créneaux économiques dans le bois, le textile et l'imprimerie. À défaut d'avoir un développement économique florissant, le milieu parvenait à se maintenir à flot. Or, à partir de 1996, une série de grands bouleversements sociaux et économiques sont venus déstabiliser l'économie locale. D’abord, l'hôpital situé à Beauceville a été fusionné à celui de la ville de Saint-George-deBeauce, dans la MRC voisine de Beauce-Sartigan et toutes les activités médicales ont été transférées vers ce grand centre urbain. Il va sans dire qu'en termes d'effectifs médicaux, cela a été un dur coup pour le milieu qui a perdu de nombreux médecins de famille. Il ne restait que ceux qui pratiquaient dans des cabinets privés.

Or, pour plusieurs raisons qui semblent relever davantage de l'ordre des conflits personnels, une certaine méfiance, voire une hostilité ouverte, s'est installée entre les médecins restants de la MRC Robert-Cliche, mais aussi entre eux et l'Agence de santé régionale et son représentant local de l'époque, le Centre de santé et de services sociaux de Beauce $(\mathrm{CSSSB})^{2}$. Chacun s'est mis à travailler sans s'occuper de ce qui se passait chez le voisin et surtout en évitant au maximum d'impliquer le CSSSB. Considérant ce climat de travail plutôt difficile, mais aussi en raison de la vétusté des cabinets de médecins existants et leur refus de s'associer au sein d'un groupe de médecine familiale (GMF), le CSSSB était incapable de convaincre les recrues potentielles en médecine de s'installer dans Robert-Cliche. Le nombre de médecins a baissé drastiquement en une décennie et en 2006, ils n'étaient plus que 14. Portrait encore plus sombre : près de la moitié de ces médecins prévoyaient prendre leur retraite dans un horizon de cinq ans. Peu à peu, de nombreux patients sont devenus orphelins et obligés de se déplacer dans la MRC voisine afin de pouvoir consulter un médecin.

Du côté du monde des affaires, cette perte de service a considérablement compliqué le repositionnement économique du milieu rendu nécessaire par les crises économiques successives du début des années 2000. Les entrepreneurs locaux étaient incapables de recruter de la main-d'œuvre spécialisée pour le développement de nouveaux projets, car les travailleurs n'étaient pas intéressés à 
venir s'installer avec leur famille dans un milieu sans services de santé décents. L'économie locale s'en est donc considérablement ressentie si bien qu'entre 2002 et 2006, la MRC Robert-Cliche était considérée comme " communauté dévitalisée » par le ministère des Affaires municipales et l'occupation du territoire (MAMOT).

Les problèmes vécus par le système de santé local étaient certes ressentis dans le milieu, mais les tenants et aboutissants étaient obscurs pour la majorité des acteurs locaux. En effet, le système de santé local ne s'est jamais associé avec d'autres instances telles que la municipalité ou le Centre local de développement (CLD) afin de trouver une solution à ses problèmes. Les médecins euxmêmes, isolés, empêtrés dans leur gestion quotidienne de la clinique et les (trop) nombreux patients qui défilaient chaque jour avaient pour la plupart abdiqué. L'un d'entre eux nous dira en ce sens: "Ce n'est pas vraiment dans le style des médecins de demander de l'aide. Toute notre vie, ça a toujours été à nous de régler les problèmes. Et je pense que ça ne nous a jamais traversé l'esprit de demander de l'aide... personne ne nous a jamais réglé nos affaires ».

Or, le problème a fini par éclater à l'automne 2006, lors des consultations citoyennes organisées par le CLD dans le cadre du programme des Pactes ruraux, eux-mêmes issus de la PNR. La population a demandé à ce que le problème d'accès aux services de santé se règle rapidement, car les jeunes familles et les personnes âgées peinaient à se déplacer dans la MRC voisine pour obtenir des services. Même son de cloche du côté du monde des affaires, qui souhaite pour sa part investir dans des projets de développement. Le CLD a pris acte de ce constat et a décidé de se positionner comme porteur de projet puisque ni l'Agence régionale, ni le CSSSB ne semblaient capables d'agir efficacement quant à ce problème.

\subsection{Une coopérative comme remède aux maux de la MRC Robert-Cliche}

Sitôt le dossier pris en main par le CLD, des consultations sont réalisées auprès des différents acteurs en santé du milieu. Très clairement, il apparait que la nouvelle structure projetée devra permettre aux médecins de se concentrer sur leur pratique médicale et laisser à d'autres le soin de faire de la gestion de la clinique et le recrutement de nouveaux collègues. D'autre part, les médecins doivent mettre leurs conflits de côté et travailler ensemble pour faciliter le succès du projet. Enfin, les cliniques médicales doivent entrer dans le $21^{\mathrm{e}}$ siècle et s'informatiser sans délai. Pour cela, le statut GMF devra être rapidement acquis.

Le modèle des coopératives de santé se démarque facilement des autres solutions, même si pour l'époque, il était encore assez peu connu. L'agente de développement déléguée au dossier va même visiter le modèle des coopératives de santé japonaises afin de s'inspirer. Elle en revient avec deux grandes orientations. D'abord, la future coopérative devra offrir ses services à l'ensemble du territoire (MRC Robert-Cliche) et non pas seulement dans la municipalité où elle sera implantée (Beauceville) afin d'éviter la compétition intermunicipalité malheureusement observée dans quelques projets semblables (Gauthier, 2012). On veut fortifier l'ensemble de la MRC et non pas affaiblir l'une ou l'autre de ses parties. Ensuite, la coopérative devra développer des services en prévention et en promotion de la santé si elle veut jouer son rôle de levier de développement social.

On réussit dans un premier temps à faire asseoir tous les médecins de MRC à la même table. Un accord tacite règne pendant quelques mois, mais les vieilles rancœurs refont surface et une partie des médecins claque la porte à tout jamais, en dépit de nombreuses tentatives ultérieures pour les ramener dans le giron du projet. De son côté, le CSSSB est d'abord rébarbatif et suspicieux vis-à-vis le CLD, qui est vu comme un organisme de développement économique qui vient s'ingérer dans les affaires de la santé. Rapidement toutefois, le CSSSB finit par adhérer complètement au projet et devient un partenaire précieux, notamment dans le dossier de la localisation de la future CS, lequel est particulièrement épineux. À peu de chose près, c'est la communauté en entier qui se mobilise afin que le projet prenne naissance. Les préfets de la MRC acceptent pour leur part de financer le démarrage du projet en réservant du Pacte rural un montant de $300000 \$$ sur six ans. Après quelques embûches de nature administrative, l'assemblée de fondation a lieu au printemps 2008. Les activités médicales, elles, commencent dès que les rénovations de la bâtisse sont terminées, soit à l'automne 2009. 


\subsection{La coopérative de santé Robert-Cliche}

Selon les statistiques les plus récentes, la Coopérative de santé Robert-Cliche (CSRC) comporte 4558 membres utilisateurs-consommateurs, 12 membres utilisateurs-producteurs (les médecins), 20 membres travailleurs (personnel administratif) et 21 membres de soutien (CSRC, 2016). Sa mission principale est de :

Développer un réseau de services de proximité en soins de santé dans la MRC RobertCliche. Elle se donne comme mandat de coordonner une offre globale de services en soins de santé sur ce territoire. Elle offre également aux citoyens un ensemble de services basés sur une approche intégrée en promotion de la santé et en prévention ainsi que des soins complémentaires (CSRC, 2013).

Le fonctionnement de la CSRC est le suivant. Elle s'occupe de la gestion d'une bâtisse aménagée en bureaux de consultation médicale. De leur côté, les médecins organisés au sein d'une société de dépense - ou dit autrement, d'une clinique médicale louent les bureaux à l'occupation plutôt que d'avoir un bail signé de 5 ou 10 ans, ce qui est avantageux pour ceux qui commencent dans la pratique. Les deux entités partagent certaines responsabilités relevant de la gestion. Ainsi, la directrice générale a droit de regard dans les deux entités, mais pas en ce qui concerne les décisions médicales afin de protéger l'autonomie professionnelle des médecins. Puisque la CSRC a acquis le statut de GMF, le CSSSB - devenu en 2015 Centre intégré en santé et services sociaux (CISSS) - fournit à la coop d'autres ressources professionnelles en santé (travailleuse sociale, infirmier spécialisé en santé publique, etc.). Ainsi donc, si la CSRC n'est, techniquement parlant, qu'une entreprise qui gère un immeuble et certains services connexes, on comprend que c'est son partenariat très serré avec la clinique médicale et le CISSS qui permet d'offrir les services médicaux de proximité dans la MRC Robert-Cliche.

Notons que pour se conformer aux lois du système de santé gratuit et universel de la province (Gouvernement du Québec, 2016) et du pays (Gouvernement du Canada, 1985), la CSRC ne peut exiger le statut de membre à quiconque afin d'avoir accès aux services de santé. Ceux-ci sont offerts à toute la population de la MRC, membres ou non-membres. Dans ces circonstances, et afin de générer des revenus autonomes, la CRSC demande dans un premier temps à ses membres une cotisation annuelle volontaire d'environ $90 \$$, ce qui est acquitté par environ $80 \%$ des membres, année après année (Tremblay, 2017). Notons que pour augmenter la part de ses revenus autonomes, la CSRC offre aussi certains autres services en santé non couverts par le régime québécois d'assurance maladie comme des services de dépistage de la tension artérielle, le monitoring de la fréquence cardiaque ou le traitement des verrues par azote liquide. Au moment de notre présence sur le terrain, la coopérative avait aussi développé des groupes de prévention et promotion en santé inspirés de ce qui se fait dans les coopératives de santé du Japon, les Hans Kaï (Bergeron et Pollender, 2014). Malheureusement, le financement obtenu par le gouvernent via les laboratoires ruraux pour implanter cette approche $(350000 \$$ pour cinq ans) n'a pas été reconduit et, faute de moyens de rendre ce service rentable, il a dû momentanément être mis sur la glace en 2015.

En dépit de ces quelques contraintes légales et financières, la coopérative de santé Robert-Cliche est toujours en phase d'expansion. Grâce au soutien de la population et des différentes instances locales, tant dans le domaine de la santé qu'à l'extérieur de celui-ci, la coopérative vise maintenant la complète autonomie financière (CSRC, 2016).

\section{Analyse du cas présenté}

Nous avons exposé dans la section précédente les raisons qui ont mené la communauté à se mobiliser afin de mettre en place la CSRC. Il nous faut revenir sur ces quelques éléments de perte de pouvoir avant de poursuivre sur la question de l'empowerment.

\subsection{La perte de pouvoir de la MRC Robert-Cliche}

Si l'on se réfère au cadre théorique de l'empowerment de Ninacs (2008), il semble que l'un des facteurs à l'origine de la perte de pouvoir de la MRC RobertCliche prend sa source au sein des communications quasi inexistantes entre les organisations en santé (cabinets de médecins, CSSSB, établissements en santé) et avec les autres acteurs de la MRC. Ces 
mauvaises communications ont empêché les acteurs de fonctionner en réseau (Brennan, Micheal et Cantrell, 2014) et peu à peu, la MRC RobertCliche est devenue incompétente au sens de Fellin (2001), car elle a «perdu [sa] capacité à s'engager dans un processus de résolution de problème afin d'atteindre [ses] objectifs [traduction libre] » (Fellin, 1995, p. 5).

Un deuxième facteur local qui a entretenu le problème pendant plusieurs années fut le manque de dispositifs de participation et d'implication citoyenne dans le système local de santé qui aurait pu initier plus tôt une mobilisation des acteurs, avec une capacité de pouvoir réelle sur les décisions (Arnstein, 1969). Il était alors difficile pour un acteur externe au domaine de la santé de s'impliquer concrètement et d'engager le milieu dans une démarche de résolution de problème.

Enfin, il semble que la perte de pouvoir de la MRC Robert-Cliche a aussi été entraînée par le disempowerment de ses organisations en santé. Le manque de communication et de travail collaboratif relevés précédemment en sont des symptômes, mais aussi, l'incapacité du CSSSB et des cabinets existant à agir concrètement sur le problème y est pour beaucoup, chacun se renvoyant la balle. Sous bien des aspects, le problème d'attraction de la MRC Robert-Cliche avait beaucoup moins à voir avec l'environnement global (services disponibles, cadre de vie, etc.) que les conditions et relations de travail des médecins (cabinets vétustes et conflits entre les médecins). Un médecin nous l'a d'ailleurs confirmé en ces termes : «Le travail, peu importe où tu es, c'est toujours la même chose, les mêmes tâches. Ce qui compte, c'est l'équipe avec qui tu es... celle avec qui tu es pris chaque jour ». C'est ainsi que, pendant plusieurs années, ce ne sont ni la population locale, ni les autorités municipales ou sociosanitaires ou encore le CLD qui se sont sentis interpellés (ou autorisés?) à agir sur un problème de nature profondément organisationnelle.

\subsection{La reprise du pouvoir}

Il fallait un porteur de projet pour initier le mouvement, et c'est exactement le rôle qu'a joué le CLD lorsqu'il a eu le mandat clair de la population pour s'occuper du problème. Au travers de notre étude, nous avons clairement établi que c'est grâce à la PNR et ses pactes ruraux, que cette impulsion a pu être donnée dans le milieu, comme cela s'est d'ailleurs vu dans de nombreuses autres collectivités (Simard et Richardson, 2014). Pour leur part, les acteurs de développement du milieu ont clairement eu une marge de manœuvre pour innover et donc, ont eu implicitement l'appui moral de l'État central, en plus de son soutien financier (300 $000 \$$ via le pacte rural pour le démarrage et 350000 \$ pour un projet déposé au programme des laboratoires ruraux).

En termes de résultats concrets, la coopérative a agi directement quant au problème d'accès à des services de santé qui s'est atténué de façon très importante pour ne devenir qu'un bruit de fond pareil à ce qui est ressenti un peu partout dans la province. De façon plus large cependant, la coopérative est devenue une nouvelle compétence collective. Pour ce faire, la CSRC a dû préalablement bâtir sa crédibilité et sa légitimité d'action, s'engager concrètement dans le milieu en fournissant des services de santé à la hauteur des besoins ressentis et se positionner comme un acteur d'importance dans l'analyse, la gestion et la réorganisation des services de santé de proximité. En termes clairs, la CSRC a dû développer son propre pouvoir d'agir (EO).

Cela étant accompli, elle a pu jouer plus adéquatement son rôle de nouvelle compétence communautaire. Elle est donc intervenue directement sur le déficit de communication et de travail en réseau préalablement observé. Cela a grandement été facilité par sa structure organisationnelle, c'est-à-dire le modèle de coopérative de solidarité. Ce type de coopérative facilite le décloisonnement des relations en impliquant au sein d'une même entité des gens ou intérêts de divers horizons : divers types de membres, leaders locaux et autres organisations de la MRC.

Nos analyses permettent aussi de faire valoir le rôle de dispositif de participation formel permanent occupé par la CSRC. En effet, la population locale peut désormais avoir un véritable pouvoir sur certaines décisions relevant de la priorisation des enjeux locaux et de l'organisation des services en santé en choisissant de devenir membre et en participant à la vie coopérative (implication bénévole, assemblée générale des membres, etc).

Sur le plan du capital communautaire, la coopérative permet aux acteurs locaux de développer un sentiment d'appartenance plus fort envers leur milieu. Cela se traduit d'abord par le membership très élevé à la coopérative (près de 5000 membres sur 
une population de près de 20000 habitants), mais aussi par la forte propension de ces derniers à s'acquitter de la cotisation annuelle volontaire, alors que celle-ci ne leur offre aucun avantage direct. Nous attribuons cela à l'éducation à la coopération et au développement local réalisé constamment par les travailleurs de la coopérative (agentes de réception), la direction et même des médecins qui travaillent dans les locaux de la CSRC. Avec cette sensibilisation s'est amorcée la transformation des préoccupations individuelles (« je n’ai pas de médecin ») vers un niveau plus collectif (je m'implique concrètement dans le développement de la communauté). C'est ce que Ninacs (2008) appelle la conscience citoyenne, premier élément du capital communautaire.

Pour stabiliser et nourrir cette conscience citoyenne, il manque un élément crucial du capital communautaire, le sentiment d'appartenance. Nous pensons que la coopérative a permis à la population locale de s'identifier positivement à la MRC Robert-Cliche, " étiquette » autrefois moins reluisante en raison des problèmes de développement socioéconomique que nous avons abordés précédemment. Pour une fois depuis bien longtemps, la MRC Robert-Cliche est devenue une communauté enviée par ses voisins, car elle a mis sur pied un projet novateur. Pour conserver cette nouvelle identité positive, la population locale est devenue prête à s'investir davantage dans le développement et le succès de la CSRC. Quelque part, dans l'histoire du projet, LA coopérative est devenue MA coopérative, pour finir par être NOTRE coopérative.

\section{Conclusion}

Notre étude a pu identifier clairement le rôle central de CSRC dans le développement du milieu. Grâce à la mobilisation des acteurs, mais aussi grâce à la compétence de ses porteurs de projet, la MRC Robert-Cliche s'est donné les moyens de se sortir de sa situation incapacitante en comblant ses déficits de participation et d'implication citoyenne, de compétences collectives, de communications et de capital communautaire. Or, nous savons aussi que ce rôle ne peut être adéquatement rempli sans que les organisations deviennent elles-mêmes compétentes et développent leur propre processus d'empowerment (EO). Toutefois, ce processus dynamique et circulaire n'a pu être initié sans la présence de conditions gagnantes : des marges de manœuvre et un soutien concret de l'État.

Nous avons identifié la PNR comme l'instance qui a facilité la naissance et le démarrage de la coopérative. Or, depuis 2015, la PNR n'est plus. Sa disparition est certainement déplorable pour les régions, car elle a démontré à de nombreuses reprises son efficacité (El-Batal et Joyal, 2015; Organisation de coopération et du développement économique, 2010; Solidarité rurale du Québec, 2013). Il ne reste qu'à espérer que le nouveau pacte fiscal, signé avec les municipalités du Québec en 2016, et le nouveau Fonds de développement des territoires (FDT) puissent jouer un rôle similaire à celui de la PNR. À tout le moins, les orientations du dernier gouvernement libéral (2015-2018) ont laissé entrevoir des possibilités intéressantes en matière de gouvernance dans le domaine des environnements favorables, du développement social et de la promotion et de la prévention en santé (Gouvernement du Québec, 2016). Mais même avec plus de responsabilités reconnues, ce sont les moyens financiers qui laissent songeurs, cela et le fait que les projets n'ont plus à être automatiquement liés à des mécanismes de consultation citoyenne (Ministère des Affaires municipales et de l'Occupation du territoire, 2015). L'avenir nous dira si ces possibilités se vérifieront et triompheront des contraintes. L'élection d'un nouveau gouvernement caquiste en 2018 et le contenu de sa plate-forme électorale ne semblent hélas pas prometteurs dans ce domaine.

\section{NOTES}

1 Pour Ninacs (2008), les organisations peuvent aussi être de petites communautés et donc, développer leur propre empowerment communautaire. Par contre, dans ce dernier cas, on ne parle pas de communautés locales, mais bien de communauté d'intérêt ou d'identité. Cela vient donc passablement compliquer l'analyse du processus d'empowerment. Dans le présent texte, spécifions que nous faisons bel et bien référence à l'empowerment communautaire de la communauté locale.

2 Notons qu'en 2015, les Agences de santé régionales ont été abolies par le projet de loi 10, Loi modifiant l'organisation et la gouvernance du réseau de la santé et des services sociaux notamment par l'abolition des agences régionales (Gouvernement du Québec, 2015), une partie des responsabilités étant transférée dans les Centres intégrés de santé et de services sociaux (CISSS ou CIUSSS), eux-mêmes ayant absorbé les CSSS. Le projet de loi est entré en vigueur le 1er avril 2015. 


\section{RÉFÉRENCES}

Arnstein, S. R. (1969). A ladder of citizen participation. American institute of Planners journal, 35(4), 216-224. Repéré à https://doi.org/10.1080/01944366908977225

Bacqué, M.-H. (2006). Empowerment et politiques urbaines aux États-Unis. Géographie, Économie, Société, 8, 107-124. Repéré à https://doi.org/10.3166/ges.8.107-124

Bergeron, C. et Pollender, H. (2014). Devenir artisan de sa santé : des communautés d'appartenance en action. Récit. Sherbrooke, QC: Observatoire québécois des réseaux locaux de services. Repéré à http://www.csssiugs.ca/c3s/data/files/Groupes_Hans_Kai_2014-07-03.pdf

Bernier, N. F. (2011). L'environnement politique de la santé. Théorie et pratique. Sainte-Foy, QC: Presses de l'Université Laval.

Boivin, I. et Fillion, L. J. (1999). La Coop-Santé Les Grès. Montréal, QC: HEC de Montréal.

Brassard, M.-J. et Darou, J. (2012). Portrait des coopératives de santé au Québec: des pouvoirs à partager. Dans Les cahiers de l'ARUC-DTC (Éd.), Recherches (Vol. 11). Lévis, QC: ARUC-DTC.

Brennan, M. A. et Israel, G. D. (2013). The power of community. Dans M. A. Brennan, J. C. Bridger et T. R. Alter (Éds), Theory, practice and community development. (pp. 78-97). New York, NY: Routledge.

Brennan, M. A. S., Micheal et Cantrell, R. K., Muthusami. (2014). Enhancing Community Through Social Interaction. IFAS Community Development. Repéré à http:// citeseerx.ist.psu.edu/viewdoc/download?doi=10.1.1.544.1439\&rep $=$ rep1\&type $=$ pdf

El-Batal, K. et Joyal, A. (2015). La Politique nationale de la ruralité québécoise relève-t-elle d'une gouvernance synergique territoriale ? Cahiers de géographie du Québec, 59(167), 189-207. Repéré à https:/ /doi.org/10.7202/1036354

Fédération des médecins omnipraticiens du Québec (2013). Rapport du groupe de travail sur l'accessibilité aux soins de santé de première ligne. Montréal, QC: Auteur. Repéré à https://fmoq-legacy.s3.amazonaws.com/fr/Presse/Dossiers/2013-10-29_ accessibilite/Rapport_acessibilite_.pdf

Fellin, P. (1995). The community and the social worker (2e éd.). Itasca, IL: Peacock publishers.

Fellin, P. (2001). Understanding american communities. Dans J. Rothman, J. Erlich et J. E. Tropman (Éds), Strategies of community intervention. (pp. 119-132). Itasca, IL: Peacock publishers.

Foucault, M. (1975). Surveiller et punir : naissance de la prison. Paris, France: Gallimard.

Friedman, J. (1992). Empowerment: the politics of alternative development. Cambridge, ON: Blackwell publishers.

Gauthier, V. (2012, 31 janvier). Trois médecins de moins à Sainte-Thècle: «C'est une catastrophe». Le Nouvelliste. Repéré à https://www.lenouvelliste.ca/archives/trois-medecins-de-moins-a-sainte-thecle-cest-une-catastrophe2094dfc8b6d849696551709cf3ac264f

Girard, J.-P. (2011). Coopératives de santé au Québec: 15 ans de cheminement, d'adaptation, d'apprentissage. I4. Repéré à http://communityrenewal.ca/sites/all/files/resource/i42011JUN23_Co-ops_sante.pdf

Girard, J.-P. (2012). Coopérative de services de santé au Québec : entre l'espoir et le doute. Vie économique, 3(4), 1-12. Repéré à http://www.eve.coop/mw-contenu/revues/15/149/RVE_vol3_no4_Girard.pdf

Gouvernement du Canada. (1985). Loi canadienne sur la santé. Ottawa: Éditeur officiel. Repéré à : https://laws-lois. justice.gc.ca/fra/lois/c-6/

Gouvernement du Québec. (2015). Projet de loi no 10 : Loi modifiant l'organisation et la gouvernance du réseau de la santé et des services sociaux notamment par l'abolition des agences régionales. Québec, QC: Éditeur officiel du Québec. Repéré à http://www.assnat.qc.ca/fr/travaux-parlementaires/projets-loi/projet-loi-10-41-1.html

Gouvernement du Québec. (2016). Accord de partenariat avec les municipalités. Québec, QC: Éditeur officiel. Repéré à https://www.mamh.gouv.qc.ca/fileadmin/publications/organisation_municipale/accord_partenariat/document_ information_accord_partenariat_municipalites.pdf 
Gourvernement du Québec. (2018). Loi sur les services de santé et de services sociaux. Québec, QC: Éditeur officiel. Repéré à http://legisquebec.gouv.qc.ca/fr/ShowDoc/cs/S-4.2

Klein, J.-L. et Champagne, C. (Éds.). (2011). Initiatives locales et lutte contre la pauvreté et l'exclusion. Québec, Québec: Presses de l'Université du Québec.

Lévesque, B. (2007). Une gouvernance partagée et un partenariat institutionnalisé pour la prise en charge des services d'intérêt général. Dans Les Cahiers du CRISES (Ếd.), Études théoriques (Vol. 0701). Repéré à https://crises.uqam.ca/wpcontent/uploads/2018/10/ET0701.pdf

Levy, C. (2012). Historique du développement et de la gouvernance locale. Dans G. Tremblay et P. F. Vieira (Éds), Le rôle de l'Université dans le développement local: expériences brésiliennes et québécoises. (pp. 63-92). Québec, Québec: Presses de l'Université du Québec.

Lukes, S. (2005). Power: a radical view (2e éd.). New York, NY: Palgrave-MacMillan.

Maynard, M. T., Gilson, L. L. et Mathieu, J. E. (2012). Empowerment—Fad or Fab? A Multilevel Review of the Past Two Decades of Research. Journal of Management, 38(4), 1231-1281. Repéré à https://doi.org/10.1177/0149206312438773

Mendell, M. (2006). L'empowerment au Canada et au Québec : enjeux et opportunités. Géographie, économie, société, 8(1), 63-85. Repéré à https://doi.org/10.3166/ges.8.63-86

Ministère des Affaires municipales et de l'Occupation du territoire. (2015). Le Fonds de développement des territoires pour appuyer les MRC dans leur compétence en développement local et régional. Muni-Express, 5. Repéré à https://www. mamot.gouv.qc.ca/publications/bulletin-muni-express/2015/n-05-23-juin-2015/

Ninacs, W. A. (2008). Empowerment et intervention - développement de la capacité d'agir et la solidarité. Québec, QC: Presses de l'Université Laval.

Organisation de coopération et du développement économique. (2010). Examens de l'OCDE des politiques rurales: Québec, Canada. Paris, France: Direction de la gouvernance publique et du développement territorial. Repéré à

http://www.oecd.org/fr/gov/politique-regionale/examensdelocdedespolitiquesruralesquebeccanada.htm\#references

Palard, J. (2009). La Beauce inc. : capital social et capitalisme régional. Montréal, QC: Presses de l'Université de Montréal.

Riger, S. (1993). What's wrong with empowerment. American Journal of Community Psychology, 21(3), 279-290. Repéré à http://dx.doi.org/10.1007/BF00941504

Rui, S. (2011). Pouvoir. Dans S. Paugam (Éd.), Les 100 mots de la sociologie. (pp. 85-86). Paris, France: Presses universitaires de France. Repéré à https:// sociologie.revues.org/1598

Simard, M. (2005). Les services de proximité en région rurale: une synthèse des connaissances. Rimouski, QC: Chaire de recherche du Canada en développement rural, Université du Québec à Rimouski.

Simard, P. et Richardson, M. (2014). Les effets de la politique nationale de la ruralité du Québec sur la santé des ruraux et des communautés. Rapports de recherche - Programme actions concertées. Québec, QC: Fonds de recherche Société et culture. Repéré à http://www.frqsc.gouv.qc.ca/documents/11326/449012/PT_SimardP_rapport_ruralite.pdf/2ff54c69-1609-4cb1-811d692893bf97ef

Solidarité rurale du Québec. (2013). Fiche d'information : Retombées économiques de la Politique nationale de la ruralité pour l'ensemble du Québec et par région. Québec, QC: Auteur. Repéré à http://www.ruralite.qc.ca/fichiers/fr/fiches_regionalisees__tout_le_qc.pdf

Tremblay, S. (2017). Processus d'empowerment communautaire dans le cadre d'un projet de développement local en santé de proximité : Le cas de la MRC Robert-Cliche et de sa coopérative de santé. (Thèse de doctorat inédite). Université du Québec à Chicoutimi. Repéré à http://constellation.uqac.ca/4385/

Vaillancourt, Y. (2017). Marges de manœuvre des acteurs locaux de développement social en contexte d'austérité. Dans Les cahiers du CRISES (Éd.), Études théoriques et méthodologiques (Vol. 1701). Repéré à https://depot.erudit.org/bitstream/004087dd/1/CRISES_ET1701.pdf

Weber, M. (1971). Économie et société : les catégories de la sociologie (Tome 1). Paris, France: Plon.

Wilkinson, K. P. (1991). The Community in Rural America. Westport: Greenwood Press.

Yin, R. K. (2009). Case study research: design and methods (4e éd.). Thousand Oaks, CA: Sage Publications. 